\title{
Does additional linear ablation after circumferential pulmonary vein isolation improve clinical outcome in patients with paroxysmal atrial fibrillation? Prospective randomised study
}

\author{
Hee-Sun Mun, Boyoung Joung, Jaemin Shim, Hye Jin Hwang, Jong Youn Kim, \\ Moon-Hyoung Lee, Hui-Nam Pak
}

Department of Cardiology, Yonsei University Health System, Seoul, Korea

\section{Correspondence to Professor Hui-Nam Pak, Department of Cardiology, Yonsei University Health System, 250 Seungsanno, Seodaemun-gu, Seoul 120-752, Republic of Korea; hnpak@yuhs.ac}

Accepted 15 December 2011 Published Online First 27 January 2012

\begin{abstract}
Objective Circumferential pulmonary vein isolation (CPVI) has been considered the cornerstone of radiofrequency catheter ablation (RFCA) for atrial fibrillation (AF).

However, it is unclear whether linear ablation in addition to CPVI improves clinical outcome.

Design Prospective randomised study to compare the efficacy of CPVI and CPVI with additional linear ablation in patients with paroxysmal AF (PAF).

Setting University hospital.

Patients This study enrolled 156 patients (male 76.3\%, $55.8 \pm 11.5$ years old (mean \pm SD)) who underwent RFCA for PAF.

Interventions CPVI ( $n=52), C P V I+$ roof line $(C P V I+R L$; $\mathrm{n}=52$ ) and $\mathrm{CPVI}+\mathrm{RL}+$ posterior inferior line (CPVI + PostBox; $n=52$ ).

Main outcome measures Procedure time, ablation time and clinical outcome.

Results (1) The CPVI group showed shorter total procedure time $(180.4 \pm 39.5 \mathrm{~min}$ vs $189.6 \pm 29.0 \mathrm{~min}$ and $201.7 \pm 51.7 \mathrm{~min}$, respectively (mean \pm SD); $\mathrm{p}=0.035)$ and ablation time $(4085.5 \pm 1384.1 \mathrm{~s}$ vs $5253.5 \pm 1010.9 \mathrm{~s}$ and $5495.0 \pm 1316.0 \mathrm{~s}$, respectively; $\mathrm{p}<0.001$ ) than the CPVI+RL and CPVI+PostBox groups. (2) During 15.6 \pm 5.0 months of follow-up, the recurrence rates 3 months after RFCA were $11.5 \%$ in CPVI, $21.2 \%$ in $\mathrm{CPVI}+\mathrm{RL}$ and $19.2 \%$ in CPVI+PostBox $(p=0.440)$. (3) The achievement rate of CPVI was $100.0 \%$, and bidirectional block rate was $80.8 \%$ in $\mathrm{CPVI}+\mathrm{RL}$ and $59.6 \%$ in $\mathrm{CPVI}+$ PostBox. The clinical recurrence rates with or without achieving bidirectional block were not significantly different from each other $(p=0.386)$. Conclusion In patients with PAF, linear ablation in addition to CPVI did not improve clinical outcome, regardless of bidirectional block achievement, while it prolonged the total procedure and ablation time.
\end{abstract}

\section{INTRODUCTION}

As atrial fibrillation (AF) begets $\mathrm{AF}^{1}$ early intervention against $A F$ at the stage of paroxysmal $A F$ (PAF) may improve clinical outcome, and radiofrequency catheter ablation (RFCA) can be considered as a first-line rhythm-control strategy in patients without any structural heart disease. ${ }^{2}$ Circumferential pulmonary vein isolation (CPVI) has been considered the cornerstone of AF catheter ablation, especially in patients with $\mathrm{PAF}^{3-9}$
However, there is still a substantial recurrence rate. To improve outcomes, an additional ablation to CPVI has been suggested to modify the substrate for $\mathrm{AF}^{10-14}$ However, ablation strategies are still under investigation, and reports of randomised trials comparing ablation strategies for PAF in structurally normal heart are limited. ${ }^{15}$ Therefore we hypothesised that an additional linear ablation to CPVI may reduce clinical recurrence of AF after RFCA. We compared three different ablation designs in a prospective randomised study: CPVI, CPVI+roof line (CPVI+RL) and CPVI+RL + posterior inferior line (CPVI+PostBox). The purpose of this study was to compare these three ablation strategies in terms of procedure time, efficacy or safety, and clinical outcome, and evaluate whether achievement of bidirectional block improves clinical outcome in patients with PAF.

\section{METHODS \\ Patient selection}

The study protocol was approved by the institutional review board of Severance Hospital of Yonsei University. All patients provided written informed consent. The study enrolled 156 patients who underwent RFCA for symptomatic drug-refractory PAF. Exclusion criteria were: (1) permanent or persistent AF; (2) left atrium (LA) size $>55 \mathrm{~mm}$ on echocardiogram; (3) associated structural heart disease other than left ventricular (LV) hypertrophy; (4) intracardiac thrombi; (5) LV ejection fraction $<45 \%$; (6) AF with rheumatic valvular disease; (7) previous AF ablation; (8) history of cardiac surgery. The patients were prospectively and randomly assigned to three groups according to the method of RFCA: CPVI alone (CPVI; $n=52$ ); linear ablation at the LA roof in addition to CPVI $(\mathrm{CPVI}+\mathrm{RL} ; \mathrm{n}=52)$; and linear ablation at the posterior inferior LA wall in addition to CPVI and RL (CPVI+PostBox; $n=52$ ). We chose the sample size on the basis of a statistical analysis to prove the superiority of CPVI with additional linear ablation, which was described in a previous study comparing ablation strategies in patients with PAF. $^{12}$ All antiarrhythmic drugs (AADs) were discontinued more than five half-lives before the procedure. Patients taking amiodarone before the procedure stopped taking it at least 4 weeks before. Anticoagulation therapy was maintained for online under the BMJ Journals unlocked scheme, see http:// heart.bmj.com/site/about/ unlocked.xhtml 
a target prothrombin time international normalised ratio (INR) of 2.0-3.0 at least 4 weeks before the procedure and continued until the day of the procedure. Both trans-thoracic echocardiography and trans-oesophageal echocardiography were performed before the procedure to determine whether patients had combined structural heart disease or LA thrombus. We imaged all patients with three-dimensional (3D) spiral CT (64 Channel, Light Speed Volume CT, Philips, Brilliance 63, Amsterdam, The Netherlands) to visually define the anatomy of the LA and pulmonary veins (PVs).

\section{Electrophysiological mapping procedure}

Intracardiac electrograms were recorded using the Prucka CardioLab Electrophysiology system (General Electric Medical Systems, Milwaukee, Wisconsin, USA), and RFCA was performed on all patients using 3D electroanatomical mapping (NavX; St Jude Medical, Minnetonka, Minnesota, USA) merged with 3D spiral CT. The high right atrium (RA), low RA and coronary sinus were mapped with a decapolar catheter (Bard Electrophysiology, Lowell, Massachusetts, USA) and duodecapolar catheter (St Jude Medical) inserted via the left femoral vein. A quadripolar catheter was also placed in the superior vena cava. Double trans-septal punctures were performed, and multiview pulmonary venograms were obtained. After trans-septal access had been secured, a circumferential PV mapping catheter (Lasso; Biosense-Webster, Diamond Bar, California, USA) was introduced with the use of a long sheath (Schwartz left 1; St Jude Medical). Systemic anticoagulation was achieved with intravenous heparin to maintain an activated clotting time of 350-400 s during the procedure. For electroanatomical mapping, the $3 \mathrm{D}$ geometry of both the LA and PV was generated using the NavX system and then merged with the 3D spiral CT images. To avoid inadvertent collateral injury, the oesophagus was visualised by barium swallow and displayed on the monitor during the ablation procedure.

\section{Radiofrequency catheter ablation}

For CPVI ablation, continuous circumferential lesions were created at the level of the LA antrum (about $2 \mathrm{~cm}$ from the PV ostia) encircling the right and left PV guided by the NavX system using an open irrigation $3.5 \mathrm{~mm}$ tip deflectable catheter (Celsius; Johnson \& Johnson, Diamond Bar, California, USA; irrigation flow rate $20-30 \mathrm{ml} / \mathrm{min} ; 30 \mathrm{~W} ; 47^{\circ} \mathrm{C}$; figure $\left.1 \mathrm{~A}\right)$. The end point of CPVI was electrical isolation of PV potentials, which was confirmed by Lasso catheter mapping during sinus rhythm, RA pacing, and/or isoproterenol infusion after $30 \mathrm{~min}$. The RL was created on the LA roof connecting the tops of two encircling lesions (irrigation flow rate $30 \mathrm{ml} / \mathrm{min} ; 35 \mathrm{~W} ; 47^{\circ} \mathrm{C}$; figure $1 \mathrm{~B})$, and achievement of bidirectional block was confirmed by pacing from the LA appendage and LA posterior wall. ${ }^{16}$ The PostBox lesion was generated by an additional posterior inferior line connecting the lower margins of the right and left CPVI lines (irrigation flow rate $25 \mathrm{ml} / \mathrm{min} ; 30 \mathrm{~W} ; 47^{\circ} \mathrm{C}$ ) to the $\mathrm{RL}$ (figure 1C), and achievement of the bidirectional block was confirmed by the absence of potential in the posterior venous atrium.

\section{Post-ablation management and follow-up}

After RFCA, the patients were followed up at the outpatient clinic at 1, 3, 6, 9 and 12 months and then every 6 months thereafter. ECG was performed at each visit. The Holter monitor $(24 \mathrm{~h}$ or $48 \mathrm{~h})$ and/or event recorder were evaluated 3, 6 and 12 months after RFCA. ${ }^{19}$ Whenever the patient reported symptoms of palpitations suggestive of arrhythmia recurrence, Holter monitor or event monitor recordings were obtained. We defined early recurrence as any episode of AF or atrial tachycardia of at least $30 \mathrm{~s}$ in duration that occurred within 3 months of the blanking period after RFCA. Warfarin was administered to all patients for at least 3 months after RFCA, targeting an INR of 2.0-3.0. All patients underwent follow-up echocardiography 12 months after RFCA or at any time the patient had cardiac symptoms. No patient was taking $\mathrm{AAD}$ at discharge after an RFCA procedure. AAD was prescribed for patients with ECG-documented AF recurrence or highly symptomatic ECG-documented frequent atrial premature beats.

\section{Data analysis}

Continuous variables are expressed as the mean \pm SD and were compared by analysis of variance. Categorical variables were compared by the $\chi^{2}$ test. When a pairwise $\chi^{2}$ test was used for three groups, the $p$ value threshold for statistical significance was adjusted to address type I error inflation for multiple comparisons ( $p$ value $<0.017$ ). The Tukey test was performed for post hoc analyses. A Kaplan-Meier analysis was used to determine the probability of freedom from arrhythmia recurrence after RFCA. $\mathrm{p}<0.05$ was considered significant.

\section{RESULTS}

\section{Patient characteristics}

The patients were prospectively and randomly assigned to undergo CPVI $(n=52)$, CPVI+RL $(n=52)$ or CPVI+PostBox $(\mathrm{n}=52)$. The mean age was $55.8 \pm 11.5$ years, and 119 patients $(76.3 \%)$ were male. Table 1 summarises patient characteristics by groups. $\mathrm{CHADS}_{2}$ score was $0.76 \pm 0.99$. The patients with hypertension accounted for $42.9 \%$, and those with diabetes $11.5 \%$. A history of ischaemic stroke was found in $7.7 \%$, and the
Figure 1 The ablation lesions for the three strategies. (A) For circumferential pulmonary vein isolation (CPVI) ablation, continuous circumferential lesions were created at the level of the left atrial (LA) antrum (about $2 \mathrm{~cm}$ from the pulmonary vein (PV) ostia) encircling the right and left PV. (B) In addition to CPVI, the roof line (RL) was created on the LA roof connecting the tops of two encircling lesions. (C) In addition to $C P V I+R L$, the PostBox lesion was generated by additional posterior inferior line connecting the lower margins of the right and left CPVI lines.
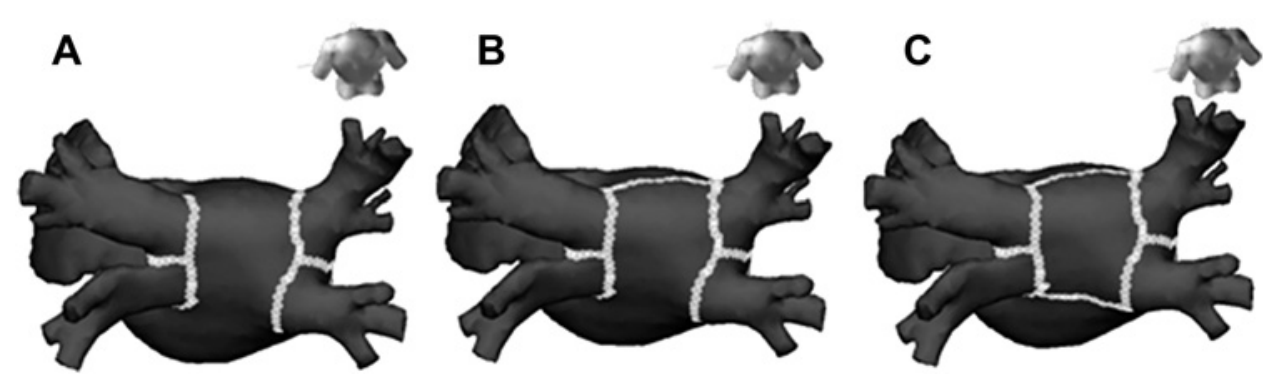
Table 1 Baseline clinical characteristics

\begin{tabular}{lllll}
\hline & $\begin{array}{l}\text { CPVI } \\
(\mathbf{n = 5 2 )}\end{array}$ & $\begin{array}{l}\mathbf{C P V I + R L} \\
(\mathbf{n}=\mathbf{5 2})\end{array}$ & $\begin{array}{l}\mathbf{C P V I + P o s t B o x} \\
(\mathbf{n = 5 2 )}\end{array}$ & $\mathbf{p ~ V a l u e}$ \\
\hline Age (years) & $54.88 \pm 12.66$ & $58.25 \pm 10.78$ & $54.27 \pm 10.62$ & 0.162 \\
Male & $37(71.2)$ & $41(78.8)$ & $41(78.8)$ & 0.567 \\
CHADS $_{2}$ & $0.71 \pm 0.94$ & $0.71 \pm 0.90$ & $0.87 \pm 1.14$ & 0.661 \\
$\quad$ Heart failure & $1(1.9)$ & $1(1.9)$ & $1(1.9)$ & 1.000 \\
$\quad$ Hypertension & $21(40.4)$ & $24(46.2)$ & $22(42.3)$ & 0.833 \\
Age $>75$ years & $2(3.8)$ & $3(5.8)$ & $0(0)$ & 0.235 \\
Diabetes & $7(13.5)$ & $3(5.8)$ & $8(15.4)$ & 0.267 \\
$\quad$ Stroke & $2(3.8)$ & $3(5.8)$ & $7(13.5)$ & 0.150 \\
LA diameter (mm) & $39.29 \pm 5.18$ & $40.42 \pm 4.28$ & $40.67 \pm 5.40$ & 0.322 \\
EF (\%) & $64.71 \pm 5.98$ & $63.79 \pm 7.63$ & $63.73 \pm 6.59$ & 0.710 \\
E/E' & $9.86 \pm 2.73$ & $9.62 \pm 3.63$ & $9.68 \pm 3.63$ & 0.936 \\
\hline
\end{tabular}

Data are expressed as number (\%) of patients or mean \pm SD.

$\mathrm{CPVI}$, circumferential pulmonary vein isolation; $\mathrm{E} / \mathrm{E}^{\prime}$, ratio of mitral valve inflow and tissue Doppler; EF, ejection fraction; LA, left atrium; PAF, paroxysmal atrial fibrillation; PostBox, additional $\mathrm{RL}$ and posterior inferior line; $\mathrm{RL}$, $\mathrm{LA}$ roof line.

mean LA anterior-posterior diameter measured by echocardiography was $40.13 \pm 4.98 \mathrm{~mm}$. The three ablation groups were well balanced with regard to demographic and baseline characteristics.

\section{Procedural results and clinical outcomes}

Table 2 summarises the procedural results and complications. The total procedure time $(\mathrm{p}=0.035)$ and radiofrequency energy delivery time $(p<0.001)$ were significantly shorter in the CPVI group than the CPVI+RL or CPVI+PostBox groups. The procedure-related complication rate was 3.8\%. Only one patient, who was in the CPVI+RL group, had a major complication and that was a transient ischaemic attack. A complication in one patient with pericardial effusion spontaneously resolved without pericardiocentesis, and complications in four patients with pericarditis were controlled by steroid (table 2). There was no difference in the complication rate among the three groups.

The early recurrence rate within 3 months of RFCA was not significantly different among the three groups $(\mathrm{p}=0.384)$. During $15.6 \pm 5.0$ months of follow-up, the clinical recurrence rate 3 months after RFCA was $11.5 \%$ in the CPVI group, $21.2 \%$ in the CPVI+RL group and $19.2 \%$ in the CPVI+PostBox group $(p=0.440 ;$ table 2$)$. Figure 2 displays Kaplan-Meier analysis of arrhythmia recurrence-free survival, and there was no significant difference among the three ablation strategies. In the ECG presentation at the time of recurrence, the proportions of atrial tachycardia were $33.3 \%$ in CPVI, $54.5 \%$ in CPVI+RL and $70.0 \%$ in CPVI+PostBox $(p=0.359)$.

\section{Complete conduction block of linear ablation and clinical outcome}

We achieved CPVI $(\mathrm{n}=52)$ in all patients $(100 \%)$, and bidirectional block in $80.8 \%$ of CPVI+RL $(n=42)$ and $59.6 \%$ of CPVI + PostBox $(\mathrm{n}=31, \mathrm{p}<0.001$, table 3$)$. The overall clinical recurrence rates with and without achievement of bidirectional block were not significantly different $(16.0 \%$ vs $22.6 \%, p=0.386)$. In patients in whom bidirectional block was achieved by linear ablations, there was no significant difference in the early recurrence rate $(21.2 \%, 28.6 \%$ and $19.4 \%$, respectively, $\mathrm{p}=0.589)$ and in the clinical recurrence rate 3 months after RFCA $(11.5 \%$, $19.1 \%$ and $19.4 \%$, respectively, $\mathrm{p}=0.978$; table 3$)$. The rate of $\mathrm{AAD}$ utilisation in patients in whom bidirectional block was achieved tended to be higher in the CPVI+RL (28.6\%) group than the CPVI (19.2\%) or CPVI+PostBox (19.4\%) groups without significance $(p=0.500)$.

\section{DISCUSSION}

In this prospective randomised comparison trial for the appropriate ablation strategy of PAF, we demonstrated that additional linear ablations to CPVI did not improve clinical outcome in spite of longer procedure or ablation time, and regardless of bidirectional block achievement. The clinical recurrence rate was also not significantly different between patients with complete conduction block by additional linear ablation and those with incomplete conduction block.

Mechanism and effectiveness of CPVI in patients with PAF

Compared with segmental ostial ablation, CPVI targets peri-PV ostial triggers or drivers, ganglionated plexi and critical mass reduction, ${ }^{17}$ as well as PV triggers. However, there is still a $10-20 \%$ recurrence rate after CPVI even in patients with

Table 2 Procedural results and clinical outcomes

\begin{tabular}{|c|c|c|c|c|}
\hline & CPVI $(n=52)$ & CPVI+RL (n=52) & CPVI+PostBox $(n=52)$ & p Value \\
\hline Total procedure time (min) & $180.43 \pm 39.52^{*}$ & $189.62 \pm 28.95$ & $201.69 \pm 51.69 *$ & 0.035 \\
\hline Ablation time (s) & $4085.5 \pm 1384.1 \dagger \neq$ & $5253.5 \pm 1010.9 \dagger$ & $5495.0 \pm 1316.0 \neq$ & $<0.001$ \\
\hline Complications & $2(3.8)$ & $1(1.9)$ & $3(5.8)$ & 0.595 \\
\hline Transient ischaemic attack & $0(0)$ & $1(1.9)$ & $0(0)$ & 0.366 \\
\hline Pericardial effusion & $1(1.9)$ & $0(0)$ & $0(0)$ & 0.366 \\
\hline Pericarditis & $1(1.9)$ & $0(0)$ & $3(5.8)$ & 0.166 \\
\hline \multicolumn{5}{|l|}{ Clinical outcomes } \\
\hline Follow-up duration (min) & $16.17 \pm 4.34$ & $15.90 \pm 4.53$ & $14.69 \pm 5.90$ & 0.273 \\
\hline ER rate & $11(21.2)$ & $18(34.6)$ & $15(28.8)$ & 0.384 \\
\hline ER rate without $A A D$ & $8(15.4)$ & $16(30.8)$ & $14(26.9)$ & 0.164 \\
\hline ER rate with $A A D$ & $3(5.8)$ & $2(3.8)$ & $1(1.9)$ & 0.595 \\
\hline Patients taking $\mathrm{AAD}$ & $18(34.6)$ & $17(32.7)$ & $22(42.3)$ & 0.560 \\
\hline CR rate & $6(11.5)$ & $11(21.2)$ & $10(19.2)$ & 0.440 \\
\hline CR rate without $A A D$ & $5(9.6)$ & $9(17.3)$ & $7(13.5)$ & 0.517 \\
\hline CR rate with $A A D$ & $1(1.9)$ & $2(3.8)$ & $3(5.8)$ & 0.595 \\
\hline Patients taking AAD & $10(19.2)$ & $14(26.9)$ & $15(28.8)$ & 0.221 \\
\hline
\end{tabular}

Data are expressed as number (\%) of patients or mean \pm SD.

${ }^{*} p=0.026, C P V I$ versus $C P V I+$ PostBox.

$+\mathrm{p}<0.001$, CPVI versus $\mathrm{CPVI}+\mathrm{RL}$.

$\neq \mathrm{p}<0.001$, CPVI versus $\mathrm{CPVI}+\mathrm{PostBox}$.

$A A D$, antiarrhythmic drug; CPVI, circumferential pulmonary vein isolation; $C R$, clinical recurrence; $E R$, early recurrence; PostBox, additional $R L$ and posterior inferior line; $R L$, $L A$ roof line. 


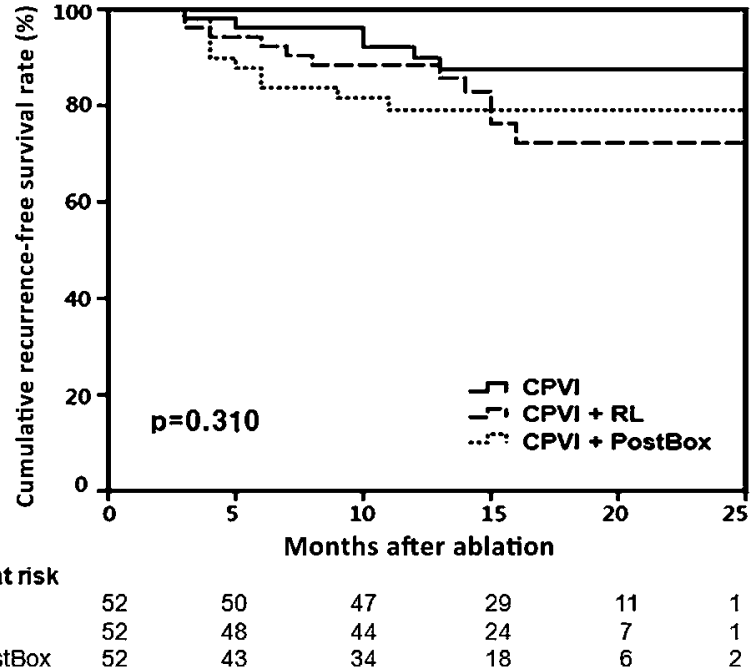

$\begin{array}{lllllll}\text { Number at risk } & & & & & \\ \text { CPVI } & 52 & 50 & 47 & 29 & 11 & 1 \\ \text { CPVI+RL } & 52 & 48 & 44 & 24 & 7 & 1 \\ \text { CPVI+PostBox } & 52 & 43 & 34 & 18 & 6 & 2\end{array}$

Figure 2 Kaplan-Meier analysis of arrhythmia recurrence-free rate in patients with paroxysmal atrial fibrillation subjected to three ablation strategies. There was no significant difference among the three ablation strategies ( $p=0.31$ by the use of the log rank (Mantel-Cox) test). CPVI, circumferential pulmonary vein isolation; PostBox, additional RL and posterior inferior line; RL, left atrial roof line.

PAF. ${ }^{19}$ Therefore additional linear ablations or complex fractionated atrial electrogram (CFAE)-guided ablation have been tried to reduce such AF recurrence after RFCA in selected patients with PAF-like long-standing persistent $\mathrm{AF}^{20-23}$ However, there was no consensus over the effectiveness of additional linear ablations to CPVI in PAF ablation. In this study, we found no additional benefit of linear ablation in addition to CPVI in patients with PAF. Therefore, to improve clinical outcome after PAF catheter ablation, we should focus on how to reduce long-term PV reconnection after initially successful PV isolation, ${ }^{24}$ not how to ablate more, at least in patients with a structurally normal heart.

\section{Why did linear ablation not change the success rate?}

In 1993, Swartz initially described a catheter-based technique for linear ablation of long-standing persistent AF (AHA abstract, Circulation 1993;90:335). ${ }^{25}$ Linear ablation eliminates more atrial substrates and partially compartmentalises the atria to prevent the formation of macro-re-entrant circuits which have been proposed to maintain $\mathrm{AF}^{16} \mathrm{~A}$ stepwise approach to add linear ablations or CFAE-guided ablation has been known to be

Table 3 Clinical outcomes in patients with achievement of bidirectional block

\begin{tabular}{lcclr}
\hline & CPVI & CPVI+RL & CPVI+PostBox & p Value \\
\hline Conduction block rate & $52(100 \%)^{*}$ & $42(80.8 \%) \dagger$ & $31(59.6 \%)^{*} \dagger$ & $<0.001$ \\
ER rate & $11(21.2 \%)$ & $12(28.6 \%)$ & $6(19.4 \%)$ & 0.589 \\
ER rate without AAD & $8(15.4 \%)$ & $10(23.8 \%)$ & $5(16.1 \%)$ & 0.538 \\
ER rate with AAD & $3(5.8 \%)$ & $2(4.8 \%)$ & $1(3.2 \%)$ & 0.871 \\
Patients taking AAD & $18(34.6 \%)$ & $12(28.6 \%)$ & $8(25.8 \%)$ & 0.666 \\
LR rate & $6(11.5 \%)$ & $8(19.1 \%)$ & $6(19.4 \%)$ & 0.978 \\
LR rate without AAD & $5(9.6 \%)$ & $6(14.3 \%)$ & $3(9.7 \%)$ & 0.739 \\
LR rate with AAD & $1(1.9 \%)$ & $2(4.8 \%)$ & $3(9.7 \%)$ & 0.279 \\
Patients taking AAD & $10(19.2 \%)$ & $12(28.6 \%)$ & $6(19.4 \%)$ & 0.500 \\
\hline
\end{tabular}

Data are expressed as number (percentages) of patients.

${ }^{*} \mathrm{p}=0.001$, CPVI versus $\mathrm{CPVI}+\mathrm{RL}$.

$t \mathrm{p}<0.001$, CPVI versus $\mathrm{CPVI}+$ PostBox.

$A A D$, antiarrhythmic drug; CPVI, circumferential pulmonary vein isolation; $E R$, early

recurrence; $L R$, late recurrence; PostBox, additional $R L$ and posterior inferior line; $R L, L A$ roof line. effective for AF substrate modification and improving clinical outcome. ${ }^{10-1426}$ While AF ablation was performed step by step, AF frequency spectra were organised as increments of linear ablation lesions and finally terminated. ${ }^{27}$ This finding suggests that stepwise reduction of atrial critical mass organises $\mathrm{AF}$, and biantral ablation and posterior wall isolation reduce critical mass by $17 \%$ and $32 \%$, respectively (unpublished data). However, critical mass reduction by linear ablation did not work in patients with PAF in this study and another non-randomised study. ${ }^{28}$ This may be because the critical mass after CPVI was too small to maintain AF in patients with less remodelled PAF, or additional linear ablation may rather potentially be proarrhythmic. ${ }^{28}$

\section{How to improve clinical outcome of PAF ablation?}

Previous studies on AF ablation have highlighted the proarrhythmic effect of incomplete and recovered gaps. ${ }^{6-8} 10-13$ Therefore, we need to find better methods of attaining durable PVI, rather than ablating more. Other biological factors that potentially contribute to conduction recovery, such as haematopoietic progenitor cell mobilisation ${ }^{29}{ }^{30}$ or myofibroblasts, ${ }^{31}$ should be considered in the future. Another point we need to consider is the definition of recurrence after AF catheter ablation. The current definition of $\mathrm{AF}$ recurrence does not consider the reduction of AF burden. ${ }^{2}$

\section{Study limitations}

This single-centre study included a relatively small number of patients. Asymptomatic and low frequent recurrence of AF may have been undetected by this study protocol. We did not quantify the change in AF burden in patients with PAF. Our ablation design for CPVI was close to PV ostial ablation. However, we do not think it would change the finding that linear ablation did not improve clinical outcome in patients with PAF with small LA. The clinical outcome of the CPVI+PostBox group may have been affected by low achievement of bidirectional block in an effort to avoid potential oesophageal injury during posterior inferior linear ablation on the LA posterior wall. Therefore further study with a larger number of patients or more intensive ECG follow-up is warranted. Because of the importance of permanent PVI and potentially proarrhythmic effects of inadvertent substrate modification, this type of randomised study would be clinically relevant for patients with persistent $\mathrm{AF}$.

\section{CONCLUSION}

We conducted the first prospective randomised comparison study for an appropriate ablation strategy for PAF and demonstrated that additional linear ablations to CPVI did not improve clinical outcome despite longer procedure or ablation time, and regardless of bidirectional block achievement.

Funding This work was supported by a grant (A085136) from the Korea Health 21 R\&D Project, Ministry of Health and Welfare and a grant (2010-0010537) from the Basic Science Research Program of the National Research Foundation of Korea under the Ministry of Education, Science and Technology of the Republic of Korea.

Competing interests None.

Ethics approval The study protocol was approved by the institutional review board of Severance Hospital of Yonsei University.

Contributors All authors contributed to the conception, design, analysis, interpretation of data and the drafting of the manuscript and revising it critically for important intellectual content. All authors are responsible for the contents and have read and approved the manuscript.

Provenance and peer review Not commissioned; externally peer reviewed. 


\section{REFERENCES}

1. Wijffels MC, Kirchhof CJ, Dorland R, et al. Atrial fibrillation begets atrial fibrillation. A study in awake chronically instrumented goats. Circulation 1995;92:1954-68.

2. Camm AJ, Kirchhof $P$, Lip GY, et al. Guidelines for the management of atrial fibrillation: the Task Force for the Management of Atrial Fibrillation of the European Society of Cardiology (ESC). Eur Heart J 2010;31:2369-429.

3. Pappone C, Rosanio $S$, Oreto $G$, et al. Circumferential radiofrequency ablation of pulmonary vein ostia: a new anatomic approach for curing atrial fibrillation. Circulation 2000;102:2619-28.

4. Oral H, Scharf C, Chugh A, et al. Catheter ablation for paroxysmal atrial fibrillation: segmental pulmonary vein ostial ablation versus left atrial ablation. Circulation 2003;108:2355-60.

5. Pappone C, Augello G, Sala S, et al. A randomized trial of circumferential pulmonary vein ablation versus antiarrhythmic drug therapy in paroxysmal atrial fibrillation: the APAF Study. J Am Coll Cardiol 2006;48:2340-7.

6. Oral H, Pappone $\mathrm{C}$, Chugh $\mathrm{A}$, et al. Circumferential pulmonary-vein ablation for chronic atrial fibrillation. N Engl J Med 2006:354:934-41.

7. Arentz T, Weber R, Burkle G, et al. Small or large isolation areas around the pulmonary veins for the treatment of atrial fibrillation? Results from a prospective randomized study. Circulation 2007;115:3057-63.

8. Ouyang F, Tilz R, Chun J, et al. Long-term results of catheter ablation in paroxysmal atrial fibrillation: lessons from a 5 -year follow-up. Circulation 2010;122:2368-77.

9. Pappone C, Rosanio S, Augello G, et al. Mortality, morbidity, and quality of life after circumferential pulmonary vein ablation for atrial fibrillation: outcomes from a controlled nonrandomized long-term study. J Am Coll Cardiol 2003:42:185-97.

10. Ernst S, Ouyang F, Lober $F$, et al. Catheter-induced linear lesions in the left atrium in patients with atrial fibrillation: an electroanatomic study. J Am Coll Cardiol 2003:42:1271-82

11. Sanders $\mathbf{P}$, Jais $P$, Hocini $M$, et al. Electrophysiologic and clinical consequences of linear catheter ablation to transect the anterior left atrium in patients with atrial fibrillation. Heart Rhythm 2004;1:176-84.

12. Hocini $\mathbf{M}$, Jais $\mathbf{P}$, Sanders $\mathrm{P}$, et al. Techniques, evaluation, and consequences of linear block at the left atrial roof in paroxysmal atrial fibrillation: a prospective randomized study. Circulation 2005;112:3688-96.

13. Willems S, Klemm H, Rostock T, et al. Substrate modification combined with pulmonary vein isolation improves outcome of catheter ablation in patients with persistent atrial fibrillation: a prospective randomized comparison. Eur Heart $J$ 2006;27:2871-8.

14. Gaita F, Caponi D, Scaglione $M$, et al. Long-term clinical results of 2 different ablation strategies in patients with paroxysmal and persistent atrial fibrillation. Circ Arrhythm Electrophysiol 2008;1:269-75.

15. Verma A, Mantovan R, Macle L, et al. Substrate and Trigger Ablation for Reduction of Atrial Fibrillation (STAR AF): a randomized, multicentre, international trial. Eur Heart J 2010;31:1344-56.

16. Pak HN, Oh YS, Lim HE, et al. Comparison of voltage map-guided left atrial anterior wall ablation versus left lateral mitral isthmus ablation in patients with persistent atrial fibrillation. Heart Rhythm 2011:8:199-206.
17. Kirchhof P, Bax J, Blomstrom-Lundquist C, et al. Early and comprehensive management of atrial fibrillation: executive summary of the proceedings from the $2 \mathrm{nd}$ AFNET-EHRA consensus conference 'research perspectives in $\mathrm{AF}^{\mathrm{\prime}}$. Eur Heart $J$ 2009:30:2969-77c

18. Everett TH 4th, Wilson EE, Olgin JE. Effects of atrial fibrillation substrate and spatiotemporal organization on atrial defibrillation thresholds. Heart Rhythm 2007;4:1048-56

19. Cappato R, Calkins H, Chen SA, et al. Updated worldwide survey on the methods efficacy, and safety of catheter ablation for human atrial fibrillation. Circ Arrhythm Electrophysiol 2010;3:32-8.

20. Kojodjojo P, O'Neill MD, Lim PB, et al. Pulmonary venous isolation by antral ablation with a large cryoballoon for treatment of paroxysmal and persistent atrial fibrillation: medium-term outcomes and non-randomised comparison with pulmonary venous isolation by radiofrequency ablation. Heart 2010;96:1379-84.

21. Miyazaki S, Kuwahara T, Kobori A, et al. Long-term clinical outcome of extensive pulmonary vein isolation-based catheter ablation therapy in patients with paroxysma and persistent atrial fibrillation. Heart 2011:97:668-73.

22. Hunter RJ, Berriman TJ, Diab I, et al. Long-term efficacy of catheter ablation for atrial fibrillation: impact of additional targeting of fractionated electrograms. Heart 2010:96:1372-8

23. Lee G, Kalman JM, Vohra JK, et al. Dissociated pulmonary vein potentials following antral pulmonary vein isolation for atrial fibrillation: impact on long-term outcome. Heart 2011:97:579-84.

24. Cappato R, Negroni S, Pecora D, et al. Prospective assessment of late conduction recurrence across radiofrequency lesions producing electrical disconnection at the pulmonary vein ostium in patients with atrial fibrillation. Circulation 2003;108:1599-604.

25. Swartz JF, Pellersels G, Silvers J, et al. A catheter-based curative approach to atria fibrillation in humans. Circulation 1994;90(suppl I):I-335.

26. Haissaguerre $\mathbf{M}$, Hocini $\mathbf{M}$, Sanders $\mathrm{P}$, et al. Catheter ablation of long-lasting persistent atrial fibrillation: clinical outcome and mechanisms of subsequent arrhythmias. J Cardiovasc Electrophysiol 2005;16:1138-47.

27. Takahashi Y, O'Neill MD, Hocini M, et al. Effects of stepwise ablation of chronic atrial fibrillation on atrial electrical and mechanical properties. J Am Coll Cardiol 2007:49:1306-14.

28. Sawhney $\mathbf{N}$, Anousheh $\mathrm{R}$, Chen $\mathbf{W}$, et al. Circumferential pulmonary vein ablation with additional linear ablation results in an increased incidence of left atrial flutter compared with segmental pulmonary vein isolation as an initial approach to ablation of paroxysmal atrial fibrillation. Circ Arrhythm Electrophysiol 2010;3:243-8.

29. Kim SK, Pak HN, Park JH, et al. Non-ischaemic titrated cardiac injury caused by radiofrequency catheter ablation of atrial fibrillation mobilizes CD34-positive mononuclear cells by non-stromal cell-derived factor-1alpha mechanism. Europace 2009;11:1024-31.

30. Shim J, Park JH, Kim JY, et al. Impaired mobilization of bone marrow derived CD34 positive mononuclear cells is related to the recurrence of atrial fibrillation after radiofrequency catheter ablation. Int J Cardiol. Published Online First: 1 June 2011. doi:10.1016/j.jijcard.2011.05.064

31. Rohr S. Myofibroblasts in diseased hearts: new players in cardiac arrhythmias? Heart Rhythm 2009;6:848-56. 